\title{
A Framework of Care in Multiple Sclerosis, Part 1
}

\section{Updated Disease Classification and Disease-Modifying Therapy Use in Specific Circumstances}

\author{
Scott D. Newsome, DO, MSCS; Philip J. Aliotta, MD, MSHA, CHCQM, FACS; \\ Jacquelyn Bainbridge, PharmD; Susan E. Bennett, PT, DPT, EdD, NCS, MSCS; Gary Cutter, PhD; \\ Kaylan Fenton, CRNP, APNP, MSCN; Fred Lublin, MD; Dorothy Northrop, MSW, ACSW; \\ David Rintell, EdD; Bryan D. Walker, MHS, PA-C; Megan Weigel, DNP, ARNP-C, MSCN; \\ Kathleen Zackowski, PhD, OTR, MSCS; David E. Jones, MD
}

\section{CME/CNE Information}

\section{Activity Available Online:}

To access the article, post-test, and evaluation online, go to http:// www.cmscscholar.org.

\section{Target Audience:}

The target audience for this activity is physicians, physician assistants, nursing professionals, and other health-care providers involved in the management of patients with multiple sclerosis (MS).

\section{Learning Objectives:}

1) Apply new information about MS to a comprehensive individualized treatment plan for patients with MS

2) Integrate the team approach into long-term planning in order to optimize rehabilitation care of patients with MS

\section{Accreditation Statement:}

This activity has been planned and implemented in accordance with the accreditation requirements and policies of the Accreditation Council for Continuing Medical Education (ACCME) through the joint providership of the Consortium of Multiple Sclerosis Centers (CMSC), Nurse Practitioner Alternatives (NPA), and Delaware Media Group. The CMSC is accredited by the ACCME to provide continuing medical education for physicians.

The CMSC designates this journal-based CME activity for a maximum of 1.0 AMA PRA Category 1 Credit/s/TM. Physicians should claim only the credit commensurate with the extent of their participation in the activity.

Nurse Practitioner Alternatives (NPA) is accredited as a provider of continuing nursing education by the American Nurses Credentialing Center's Commission on Accreditation.

NPA designates this enduring material for 1.0 Continuing Nursing Education credit.

Laurie Scudder, DNP, NP, has served as Nurse Planner for this activity. She has disclosed no relevant financial relationships.

\section{Disclosures:}

Francois Bethoux, MD, Editor in Chief of the International Journal of MS Care (IMSC), has served as Physician Planner for this activity.
He has received royalties from Springer Publishing and has received intellectual property rights from Biogen.

Laurie Scudder, DNP, NP, has served as Nurse Planner for this activity. She has disclosed no relevant financial relationships.

Scott D. Newsome, DO, MSCS (author), has served on scientific advisory boards for Biogen, Genentech, Novartis, and Genzyme, and has performed contracted research (institution received funds) for Biogen, Genentech, and Novartis.

Philip J. Aliotta, MD, MSHA, CHCQM, FACS (author), has served on speakers' bureaus for Astellas Pharma, Actavis, Augmenix, and Allergan and has performed contracted research for Allergan.

Jacquelyn Bainbridge, PharmD (author), has disclosed no relevant financial relationships.

Susan E. Bennett, PT, DPT, EdD, NCS, MSCS (author), has served on speakers' bureaus for Acorda Therapeutics, Biogen, and Medtronic; has received consulting fees from and performed contracted research for Acorda Therapeutics; and is chair of the Clinical Events Committee at Innovative Technologies.

Gary Cutter, PhD (author), has participated on Data and Safety Monitoring Committees for AMO Pharma, Apotek, Gilead Pharmaceuticals, Horizon Pharmaceuticals, Modigenetech/Prolor, Merck, Merck/Pfizer, Opko Biologics, Neuren, Sanofi-Aventis, Reata Pharmaceuticals, Receptos/Celgene, Teva Pharmaceuticals, NHLBI (Protocol Review Committee), and NICHD (OPRU Oversight Committee); has received consulting fees from and/or served on speakers' bureaus and scientific advisory boards for Cerespir, Genzyme, Genentech, Innate Therapeutics, Janssen Pharmaceuticals, KleinBuendel Incorporated, Medlmmune, Medday, Nivalis, Novartis, Opexa Therapeutics, Roche, Savara, Somahlution, Teva Pharmaceuticals, Transparency Life Sciences, and TG Therapeutics; and is President of Pythagoras, Inc., a private consulting company located in Birmingham, AL.

Kaylan Fenton, CRNP, APNP, MSCN (author), has disclosed no relevant financial relationships. 
Fred Lublin, MD (author), has received consulting fees/fees for nonCME/CE activities from Bayer HealthCare Pharmaceuticals, Biogen, EMD Serono, Novartis, Teva Neuroscience, Actelion, Sanofi/Genzyme, Acorda, Questcor/Mallinckrodt, Roche/Genentech, Medlmmune, Osmotica, Xenoport, Receptos/Celgene, Forward Pharma, Akros, TG Therapeutics, AbbVie, Toyama, Amgen, Medday, Atara Biotherapeutics, Polypharma, Pfizer, Johnson \& Johnson, Revalesio, Coronado Bioscience, and Bristol-Myers Squibb; has served on speakers' bureaus for Genentech/Roche and Genzyme/Sanofi; has performed contracted research for Acorda, Biogen, Novartis, Teva Neuroscience, Genzyme, Xenoport, and Receptos; is the co-chief editor of Multiple Sclerosis and Related Disorders; and has an ownership interest in Cognition Pharmaceuticals.

Dorothy Northrop, MSW, ACSW (author), has disclosed no relevant financial relationships.

David Rintell, EdD (author), has received consulting fees from Novartis and has served as a patient education speaker for Teva Neuroscience. He started as a salaried employee of Sanofi Genzyme in November 2015. Dr. Rintell's work on this project was completed before he became a salaried employee of Sanofi Genzyme.

Bryan D. Walker, MHS, PA-C (author), has served on scientific advisory boards for EMD Serono and Sanofi Genzyme and owns stock in Biogen.

Megan Weigel, DNP, ARNP-C, MSCN (author), has received consulting fees from Mallinckrodt, Genzyme, and Genentech, and has served on speakers' bureaus for Bayer Corp, Acorda Therapeutics, Teva Neuroscience, Biogen, Mallinckrodt, Genzyme, Novartis, and Pfizer.

Kathleen Zackowski, PhD, OTR, MSCS (author), has performed contracted research for Acorda Therapeutics.

David E. Jones, MD (author), has received consulting fees from Biogen and Novartis, and has performed contracted research for Biogen.

One anonymous peer reviewer for the IJMSC has performed contracted research (institution received funds) for Novartis, Chugai, and Biogen. Another reviewer has received consulting fees and served on speakers' bureaus for Biogen, Sanofi Genzyme, Genentech, EMD Serono, and Novartis. The third reviewer has disclosed no relevant financial relationships.

Lori Saslow, MS (medical writer), has disclosed no relevant financial relationships.

The staff at the IJMSC, CMSC, NPA, and Delaware Media Group who are in a position to influence content have disclosed no relevant financial relationships.
Note: Disclosures listed for authors are those applicable at the time of their work on this project and within 12 months previously. Financial relationships for some authors may have changed in the interval between the time of their work on this project and publication of the article.

\section{Funding/Support:}

Funding for the Framework of Care consensus conference was provided by the Consortium of Multiple Sclerosis Centers, Mallinckrodt Pharmaceuticals, and Mylan Pharmaceuticals.

\section{Method of Participation:}

Release Date: December 1, 2016

Valid for Credit Through: December 1, 2017

In order to receive CME/CNE credit, participants must:

1) Review the $C M E / C N E$ information, including learning objectives and author disclosures.

2) Study the educational content.

3) Complete the post-test and evaluation, which are available at http://www.cmscscholar.org.

Statements of Credit are awarded upon successful completion of the post-test with a passing score of $>70 \%$ and the evaluation.

There is no fee to participate in this activity.

\section{Disclosure of Unlabeled Use:}

This CME/CNE activity may contain discussion of published and/ or investigational uses of agents that are not approved by the FDA. CMSC, NPA, and Delaware Media Group do not recommend the use of any agent outside of the labeled indications. The opinions expressed in the educational activity are those of the faculty and do not necessarily represent the views of CMSC, NPA, or Delaware Media Group.

\section{Disclaimer:}

Participants have an implied responsibility to use the newly acquired information to enhance patient outcomes and their own professional development. The information presented in this activity is not meant to serve as a guideline for patient management. Any medications, diagnostic procedures, or treatments discussed in this publication should not be used by clinicians or other health-care professionals without first evaluating their patients' conditions, considering possible contraindications or risks, reviewing any applicable manufacturer's product information, and comparing any therapeutic approach with the recommendations of other authorities.

From the Division of Neuroimmunology and Neuroinfectious Diseases, The Johns Hopkins Hospital, Baltimore, MD, USA (SDN, KF); Western New York Urology Associates, Buffalo, NY, USA (PJA); Department of Neurology, University of Colorado, School of Medicine, and Department of Clinical Pharmacy, Skaggs School of Pharmacy and Pharmaceutical Sciences, Aurora, CO, USA (JB); Departments of Rehabilitation Science, Neurology, and Neurosurgery, State University of New York at Buffalo, Buffalo, NY, USA (SEB); Department of Biostatistics, UAB School of Public Health, University of Alabama at Birmingham, Birmingham, AL, USA (GC); Department of Neurology, The Corinne Goldsmith Dickinson Center for Multiple Sclerosis, Icahn School of Medicine at Mount Sinai, New York, NY, USA (FL); Comprehensive MS Care Center at Holy Name Hospital, Teaneck, NJ, USA (DN); Harvard Medical School, Boston, MA, USA, and Partners MS Center, Brigham \& Women's Hospital, Partners Pediatric MS Center, Massachusetts General Hospital, Brookline, MA, USA (DR); Department of Neurology, Division of MS and Neuroimmunology, Duke University Medical Center, Durham, NC, USA (BDW); Baptist Neurology, Jacksonville Beach, FL, USA (MW); Kennedy Krieger Institute, Departments of Physical Medicine \& Rehabilitation and Neurology, Johns Hopkins University School of Medicine, Baltimore, MD, USA (KZ); and University of Virginia Health System, Charlottesville, VA, USA (DEJ). Ms. Fenton is now at the Center for Neurological Disorders, Milwaukee, WI, USA. Dr. Rintell is now at Global Patient Advocacy, Rare Diseases, Sanofi Genzyme, Brookline, MA, USA. Correspondence: Scott D. Newsome, DO, Division of Neuroimmunology and Neuroinfectious Diseases, The Johns Hopkins Hospital, 600 N. Wolfe St., Pathology 627, Baltimore, MD 21287; e-mail: snewsom2@jhmi.edu. 
The Consortium of Multiple Sclerosis Centers brought together a multidisciplinary group of clinicians and researchers to explore and evaluate current standards in multiple sclerosis (MS) care. This Framework Taskforce comprised 13 clinician-experts from varying fields, including neurology, biostatistics, MS nursing, pharmacy, physician assistants, rehabilitation specialists, psychology, social work, and urology. The methods of this initiative included analysis of a needs assessment survey and an extensive literature review. The outcome is a two-part continuing education series reviewing best practices on specific key topics in MS care. Part 1, presented herein, discusses the background of MS care and focuses on the disease-modifying therapies (DMTs) currently approved by the US Food and Drug Administration for use in MS. Best practices emphasize a comprehensive, multidisciplinary, patient-centered team approach. Included are suggestions for effective communication among health-care team members. Up-to-date information is provided on the clinical course of MS, including current disease-specific terminology, which is of utmost importance in identifying optimal treatments for people with MS. Specific circumstances that health-care providers may encounter are presented, including methods for selecting a DMT, when to switch therapies, and treatment and evaluation considerations when a suboptimal response to therapy occurs. In addition, standardized magnetic resonance imaging is important for diagnosis and follow-up. Magnetic resonance imaging is recommended before starting medication therapy or switching DMTs; new lesions suggest the need to confirm adherence or consider advancing therapy. Shared decision making among health-care providers and people with MS is encouraged. Int J MS Care. 2016;18:314-323.

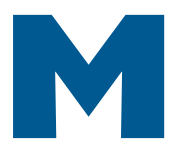

ultiple sclerosis (MS) is a common neurologic disease of the central nervous system that often affects young adults in the prime of their lives. The disease is the most common cause of nontraumatic disability in people aged 10 to 65 years. ${ }^{1}$ Owing to the multiple symptoms of MS, this disabling neurologic disease has an unpredictable and varied effect on a person's physical and cognitive abilities, emotions, activities of daily living, and quality of life. In addition, MS is an expensive disease to treat and can be financially burdensome for people with MS and their families and for insurers and payers; national annual costs of MS are estimated to be $\$ 6.8$ billion to $\$ 11.9$ billion. $^{2}$

In April 2015, the Consortium of Multiple Sclerosis Centers (CMSC) convened the first of two in-person meetings of a multidisciplinary group of clinicians and researchers to explore the current MS standards of care. This meeting was a continuation and offshoot of the concerns expressed at a 1993 conference sponsored by the CMSC. ${ }^{3}$ The Framework Taskforce comprised 13 clinician-experts from varying fields, including neurology, biostatistics, MS nursing, pharmacy, physician assistants, rehabilitation specialists, psychology, social work, and urology. This group was tasked with the goal of reviewing the MS literature and current standards of care and developing an up-to-date, evidence-based Framework of Care for clinicians on the front line of MS care. These multidisciplinary experts plan to continue with future meetings to further develop additional aspects of the MS Framework of Care.

The planned outcome of the meeting was to provide a concrete tool for all MS health-care providers. The result is a two-part series of continuing education articles focusing on the specific knowledge gaps identified in a needs assessment survey conducted with the CMSC membership in 2014. Reflecting the identified needs, Part 1 discusses MS disease characteristics and disease-modifying therapies (DMTs), including selecting therapies, identifying a suboptimal treatment response, and switching therapies. Part 2 focuses on MS symptoms (not included herein); survey participants expressed strong interest in detailed information about symptomatic care for bladder and bowel dysfunction, mental health issues, and cognitive impairment. To address specific issues described in the survey, the Framework Taskforce decided to concentrate on the subspecialty care related to bladder, bowel, mental health, and cognition, and these areas of symptomatic care are covered in Part 2 of the series. Rehabilitation was also identified as an important topic of interest and is discussed at the conclusion of Part 2.

\section{Background}

Before 1993, symptomatic management was the focus of MS care because there were several medications used successfully but no DMTs. In the early 1990s, DMTs became available, and as of May 2016, there were 14 DMTs approved by the US Food and Drug Administra- 
tion for use in MS.4-6 It is widely believed that there is now a strong emphasis on DMTs and a minor focus on symptom management in clinical practice.

People with MS are appropriately managed by effectively addressing the course of the disease and its resultant symptoms. A multidisciplinary focus on symptoms is highlighted in the recently published American Academy of Neurology MS quality measures, in which Rae-Grant et al. ${ }^{7}$ discuss gaps in care and areas where quality measures may be used to improve the care of people with MS. The future of quality MS care requires finding a balance in which disease and symptoms are co-managed equally; indeed, the merit-based incentive payment system, as defined in the Medicare Access and CHIP Reauthorization Act of 2015, may demand it.

Current practice should emphasize a comprehensive care model in MS, incorporating a patient-centered approach with an organized, multidisciplinary plan of care combined with an experienced team of health-care professionals (Figure 1). Each specialist brings expertise and knowledge to meet the varied needs of patients and their families; however, not every patient may need to consult with every subspecialty. All care providers working together as a team will provide the most benefit to people with MS. Above all, the entire MS team should strive to see patients as people who happen to have MS, not as MS patients. It is a subtle difference but can have a huge effect on how they perceive themselves and function.

Effective communication between team members is essential to optimize efforts to address the medical, social, vocational, emotional, and educational needs of people with MS. Recognized MS organizations, including the Multiple Sclerosis Association of America (http://mymsaa.org), Multiple Sclerosis Coalition (http://www.ms-coalition.org), Multiple Sclerosis Foun- dation (http://www.msfocus.org), and National Multiple Sclerosis Society (http://www.nationalmssociety. org), are important sources of information, support, and assistance to patients and their families. Support groups may be helpful to some, and these organizations help facilitate these outlets for social events and activities.

An effective team approach allows for coordinated services and continuity of care, which focuses on the best interests of people with MS and their families. ${ }^{1}$ Unfortunately, the reality is that the coordination of large medical teams can be challenging, especially in rural areas where specialty services may not exist. To achieve optimal results, the team approach must emphasize the importance of communication, not only between health-care providers and patients but also between the various providers on the treatment team itself. When the health system facility and various providers use the same electronic health record (EHR), some communication is manageable. Although the EHR may assist with communication within the facility and between providers, communication is still a challenge across different EHR 
platforms. For effective, multidisciplinary care, it can be helpful to define each team member's role and how they will communicate with one another.

An important example of effective communication in a collaborative environment occurs when any member of the team identifies a patient experiencing a relapse. For example, the patient's physical therapist notices weakness that was not evident in previous weeks; he or she then contacts the neurologist to express concern about the patient or the effect of a symptomatic medication change. This type of communication between care providers will positively affect the care of the patient, resulting in earlier detection and management of the relapse. This effective care model is possible only when all health-care providers on the team have an organized network of communication and a solid understanding of the disease process, diagnosis, and treatment options.

The team approach may be achieved through a variety of different strategies. The MS care team may be part of a coordinated group in an MS care center or may include specialists from the same hospital or facility. Alternatively, the team may include single practitioners in a local community who refer patients to the varied specialists that they come to know professionally to provide optimum care to their patients with MS, in which case the patient coordinates input from the specialists. An effective team approach is possible only if the clinician can call on a selected group of reliable, knowledgeable providers. Some providers elect to obtain certification in MS care as an MS Certified Specialist (MSCS), an MS Certified Nurse (MSCN), or Certification in Rare Neuroimmunologic Disorders (CRND). These credentials tell health-care providers, patients, their families, and others that the clinician has targeted knowledge and expertise in the care of people with MS.

\section{Clinical Course of MS and Terminology}

The course of MS is variable, and $85 \%$ to $90 \%$ of people with MS begin with relapsing-remitting disease. A high proportion of people with relapsing-remitting MS eventually transition to secondary progressive MS, determined by progressive accumulation of disability with few or no relapses or new lesions on magnetic resonance imaging (MRI). Approximately $10 \%$ to $15 \%$ of individuals with MS present with a primary progressive disease course, which consists of progression of disability from initial symptom onset (typically spastic monoparesis/hemiparesis) without clinical relapses. ${ }^{1,8}$ When managing people with MS, it is important to determine the presence of inflammatory activity by clinical or MRI criteria. There may also be disease progression that occurs in the absence of measurable inflammation (ie, neurodegeneration).

Based on recently updated clinical criteria, ${ }^{9}$ providers are encouraged to identify patients as having relapsing or progressive MS, with inflammatory versus noninflammatory (ie, active vs. nonactive) disease, and to determine whether patients have disease progression independent of relapses. The term worsening is preferred to progressing to describe patients with relapsing disease whose symptoms of disability are accumulating due to frequent relapses or incomplete recovery from relapses. The term disease progression is reserved for those in a progressive phase with evidence of gradual worsening over time. ${ }^{9}$

Active disease can be measured by clinical or MRI activity. Active clinical disease may be determined by the presence of relapses, which are acute or subacute episodes of new or increasing neurologic dysfunction followed by full or partial recovery in the absence of fever or infection. To meet the criteria for an MS relapse, a new or worsening neurologic symptom must persist for at least 24 hours. ${ }^{9}$ An MS relapse may include blurred vision, diplopia, weakness or numbness in an extremity or the trunk, vertigo, ataxia, bladder/bowel dysfunction, significant cognitive changes, fatigue, and so on; the presentation may vary from patient to patient and from relapse to relapse. In a 2003 study, Lublin et al. ${ }^{10}$ evaluated disability in more than 200 patients before and after relapse using the Expanded Disability Status Scale (EDSS). Almost $50 \%$ of people had at least a 0.5 -point increase in their EDSS score after a relapse. One-third of patients had sustained disability over time from a single relapse. Each relapse can leave residual deficits even after symptoms have resolved. ${ }^{10,11}$ Relapse frequency, interval between relapses, location of lesion, and poor recovery from relapses can affect disease worsening. ${ }^{12,13}$ The MRI criteria for active disease include the occurrence of contrast-enhancing $\mathrm{T} 1$ or new or unequivocally enlarging T2 hyperintense lesions. ${ }^{9}$

In addition to relapsing and progressive MS, clinically isolated syndrome (CIS) is considered part of the spectrum of MS phenotypes, especially those with an MRI showing lesions consistent with demyelination. Abnormal MRI findings suggestive of demyelination place a patient with CIS at higher risk of going on to 
develop clinically definite MS. ${ }^{14}$ Radiologically isolated syndrome (RIS) is the incidental finding of MRI lesions suggestive of demyelination without clinical symptoms raising suspicion of MS. To date, RIS is not considered an MS phenotype because patients do not demonstrate clinical signs and symptoms; however, recent data ${ }^{15}$ suggest that $34 \%$ of patients with RIS will experience a clinical event within 5 years. Moreover, Okuda et al. ${ }^{15}$ demonstrated that asymptomatic spine lesions in RIS indicate a higher risk of MS independent of brain lesions shown on MRI.

A variety of factors are associated with a more aggressive phenotype of MS. Clinical factors associated with aggressive MS include male sex, older age at onset, African American descent, initial involvement of the motor or cerebellar region, sphincter involvement, frequent relapses especially in the first 2 years after diagnosis, poor recovery from relapses, and multifocal involvement at the onset of disease. Paraclinical factors include a high lesion burden on MRI, brain atrophy, and a low vitamin D level. ${ }^{13,16}$

\section{DMTs: Selecting Therapies, Identifying a Suboptimal Treatment Response, and Switching Therapies}

As of May 2016, 14 US Food and Drug Administration-approved DMTs were available for the treatment of relapsing forms of MS; several are also approved for CIS. There are injectable, intravenous, and oral DMTs, and these medications have different mechanisms of action to effectively reduce the inflammatory component of the disease. This is accomplished by reducing relapses, reducing new MRI activity, and, hopefully, delaying long-term disability progression. At the present time, there are no formal recommendations and no generally accepted algorithms for the selection of DMTs. ${ }^{17}$ The Framework Taskforce encourages clinicians to consider an individualized approach to selecting the appropriate therapy for people with MS and to continue with a focus on individualized therapy goals when considering switching and escalating therapies (ie, shared decision making).

When selecting the most appropriate DMT for a patient, there are a multitude of factors to consider, including lifestyle, availability of a care partner, acceptability of injection or availability of infusion services, mental health concerns, future plans regarding pregnancy, and comorbidities of the patient. ${ }^{17}$ There are also
DMT considerations, including medication efficacy, mechanisms of action, adverse effects, schedule of treatment, ease and route of administration, and previous use of DMTs. The severity of a patient's MS should be considered; more aggressive treatment may be appropriate in patients with risk factors for more severe disease. Although comparator trials are limited, it is thought that the newer medications may have improved efficacy over first-generation DMTs.

Treating this disease early, within the first 5 years of clinical symptom onset, seems to be very important. A variety of studies have shown that MS inflammatory activity seems to be most robust early on in the disease and that DMTs seem to be more effective against the inflammatory phase of the disease. Tintore et al. ${ }^{18}$ identified early factors in CIS that are associated with longterm disability. Specifically, the presence of oligoclonal bands, new T2 lesions, and incomplete recovery from relapses are independent predictors of disability accumulation. The baseline lesion load, development of new lesions during the first year, and not starting a DMT before a second attack are independent predictors of further relapses. Evaluating people with MS for these parameters early on can help determine how aggressive to be with DMTs.

Because MS is heterogeneous, an individual may not experience an optimum clinical response to every therapy. ${ }^{17}$ Note that some DMTs require laboratory monitoring. Information about monitoring parameters is available in the prescribing information for each DMT. Some clinicians will conduct closer monitoring for some laboratory studies depending on the medication, comorbidities, and so on. In many practices, an anti-JC virus antibody is obtained when a new patient is diagnosed as having MS, before medication initiation.

Best practice algorithms for clinicians were published in the October 2014 Special Supplement of the International Journal of MS Care. ${ }^{19}$ Helpful guidelines for decision making are provided regarding therapeutic selection in patients with relapsing-remitting MS, CIS, and aggressive onset or early poor prognostic indicators of MS. The supplement also reviewed switching DMTs owing to lack of efficacy. The supplement may be viewed at http://ijmsc.org/toc/ijmc/16/S6.

For patients taking natalizumab, the risk of progressive multifocal leukoencephalopathy (PML) should be considered. The risk is stratified based on three established risk factors: JC virus seropositivity, use of previous 
immunosuppression, and duration of therapy. Patients taking natalizumab should be monitored for anti-JC virus antibodies at least every 6 months, and index levels may help further stratify the risk of PML in JC virusseropositive patients. ${ }^{20}$ After patients initiate natalizumab therapy, they should be monitored for PML with frequent clinical and radiologic follow-up, the specific timing of which will depend on risk stratification.

Magnetic resonance imaging is recommended before starting medication therapy or switching to another DMT and at specific intervals over time to assess treatment response. Revised recommendations for a standardized MRI protocol and clinical guidelines for the diagnosis and follow-up of MS are available. ${ }^{21,22}$ A summary of these recommendations is shown in Table 1.

No evidence of disease activity (NEDA) $)^{23,24}$ is an evolving concept. The goal of NEDA for patients receiving a DMT is that there are no new or enlarging T2 lesions, no new gadolinium-enhancing lesions, no relapses, and no confirmed worsening of the EDSS score. Some consider adding a fourth element, brain atrophy, but this measure is frequently not available in clinical practice, and gauging excess atrophy is often difficult. A reasonable goal is to control disease activity and prevent irreversible damage as quickly and effectively as possible. ${ }^{4}$ Although NEDA is a reasonable concept clinically, developing standards is difficult because NEDA depends heavily on the frequency of MRI evaluations.

When should clinicians consider switching therapy? Based on NEDA data, ${ }^{25-29}$ it may be advisable to have a "zero tolerance policy." It is important not to wait until disability accrues to make the decision to switch.

\section{Table 1. Timing of the core brain MRI protocol with gadolinium for patients with an established diagnosis of $\mathbf{M S}^{21}$}

- Before starting or switching disease-modifying therapy

- Approximately 6 mo after switching disease-modifying therapy to establish a new baseline with the new therapy

- Every 1-2 y while receiving disease-modifying therapy to assess for subclinical disease activity

- Unexpected clinical deterioration or reassessment of original diagnosis

- Note: Routine spinal cord follow-up is not required unless the syndrome is predominantly recurrent transverse myelitis

- Timing of PML surveillance brain MRI protocol: every 12 mo for serum JC virus antibody-negative patients

- Every 3-6 mo for serum JC virus antibody-positive patients and $\geq 18$ mo of natalizumab therapy

Abbreviations: MRI, magnetic resonance imaging; MS, multiple sclerosis; PML, progressive multifocal leukoencephalopathy.
The Taskforce suggests that clinicians consider switching when breakthrough disease activity is apparent, as evidenced by definite relapses, changes that may be suggestive of disability progression, and substantive MRI activity (new/enlarging T2 lesions or gadoliniumenhancing lesions) even when the patient is asymptomatic. Prosperini et al. ${ }^{30}$ demonstrated that patients with subclinical MRI disease activity after 1 year of treatment with interferons were at greatest risk for future disability progression. In addition, developing asymptomatic brain and spine lesions are a risk factor for future relapses. ${ }^{31}$

If a patient is having breakthrough disease, it is important to monitor treatment adherence and to consider intolerable medication adverse effects, risk tolerance, and JC virus antibody seroconversion. It is also important to check neutralizing antibodies in patients taking interferon and to check anti-natalizumab antibodies for patients taking natalizumab. Retrospective studies have shown that switching therapies after breakthrough disease may lead to favorable responses. One analysis showed that switching from an injectable immunomodulatory medication to fingolimod was associated with fewer relapses and better disability outcomes compared with a switch to another injectable medication. ${ }^{32}$ Another study suggested that switching to natalizumab from an injectable DMT may be more effective than switching to fingolimod regarding relapse rate and shortterm disability. ${ }^{33}$

Although there are no class I data for changing therapy in the setting of a suboptimal response, it seems appropriate to try to achieve a better response because DMTs are preventive and not restorative. Coyle et al. ${ }^{34,35}$ identified patients with a suboptimal response as defined by clinical evidence of disease activity and concerning levels of MRI activity. Clinical evidence of disease activity includes at least two relapses within 1 year or one significant relapse in the past year. Concerning levels of MRI activity include substantial MRI changes at 1 year in the absence of clinical symptoms or continued MRI activity on serial MRIs. ${ }^{34,35}$ Another algorithm for treatment optimization in MS is outlined by Freedman et al. ${ }^{16} \mathrm{~A}$ recent consensus opinion among US neurologists demonstrated a more aggressive approach to treating MS early in the course of the disease than what was previously recommended. Many consider switching DMTs if at least two new T2 lesions or at least one new gadolinium-enhancing lesion occurs when patients are undergoing therapy. ${ }^{36}$ 
When switching therapies, it is reasonable to consider medications with a different mechanism of action. In addition, it is important to take into consideration the medication's adverse effect profile and specifics required to monitor patients receiving DMTs. It is also important to look at the consequences of escalating therapy and to consider the potential effects that previous agents may have on increasing the future risk of severe infections such as PML. Other factors to consider include the patient's risk of clinical worsening based on clinical characteristics, MRI characteristics, the likelihood that the new treatment will limit disease progression, the relative safety of the new treatment, and patient preferences regarding treatment and risks. Also consider increased patient responsibility because newer DMTs require more monitoring; if a patient consistently misses appointments or is not compliant with needed laboratory work, then the newer DMTs that require monitoring may have greater risk than benefit.

An escalation paradigm is often used to help minimize both medication risks and long-term disability in MS, although clinicians' and patients' thresholds to switch and escalate therapies have become lower due to the expanding armamentarium of MS DMTs. Older injectable therapies, either glatiramer acetate or an interferon, are still often started as first-line therapy and continued for at least 6 months to assess an adequate treatment response, although starting with an oral or infusion DMT is becoming a more popular treatment approach. It is in the best interest of the patient to be monitored closely for a suboptimal treatment response (ie, breakthrough relapse or new MRI lesions) because if a patient is deemed to have breakthrough disease activity, switching and escalating therapy should be considered. If there is a suboptimal treatment response in a patient receiving an injectable therapy who is not deemed to have aggressive disease, another injectable therapy may be considered, especially in individuals with lower risk tolerance. If there is more concern for aggressive disease and the patient is receiving an injectable therapy, switching to an oral therapy (fingolimod, dimethyl fumarate) or an infusion therapy (natalizumab) should be strongly considered. If the suboptimal response continues, third-line agents such as alemtuzumab may be considered.

In contrast to the aforementioned escalation paradigm, some clinicians consider using an induction paradigm, which starts with a highly effective agent, perhaps prioritizing efficacy over safety. Arnold et al. ${ }^{37}$ demonstrated that glatiramer acetate after low-dose mitoxantrone induction may be an effective alternative, although the clinical trials of mitoxantrone arguably underestimated its risk of cardiotoxicity and leukemia. Although its label suggests that it should be reserved as a third-line agent, the durable effect noted with alemtuzumab supports its use as an induction therapy. Experimental/ unproven therapies or off-label therapies (ie, rituximab, cyclophosphamide) may also be considered. ${ }^{16}$ Some consider natalizumab first-line therapy in JC virus-seronegative patients, especially if an individual presents with an aggressive phenotype of MS, although the risk of breakthrough disease after natalizumab cessation really is not congruent with the concept of an induction agent.

Shared decision making among people with MS and health-care providers is encouraged, and partners should be included in the conversation when treatment decisions are made. Partners and close family members experience the consequences of treatment decisions and should have a voice in these discussions, with prior patient permission, of course. In addition, because MS is associated with a substantial financial burden related to direct and indirect costs, the ability of patients to bear the expense of medication, medical services, and care should be considered when deciding on the best course of treatment for a patient. There are costs that

\section{Prectice Points}

- Today, there are more than a dozen diseasemodifying therapies (DMTs) approved by the US Food and Drug Administration for use in MS.

- Health-care providers should emphasize a comprehensive care model when caring for people with MS by incorporating a patient-centered approach with an organized, multidisciplinary plan of care combined with an experienced team of health-care professionals.

- It is important to initiate therapy for MS early in the course of the disease, within the first 5 years of clinical symptom onset, and to consider switching therapies when there is evidence of new demyelinating disease activity.

- Magnetic resonance imaging is recommended before starting a medication or switching to another DMT and at specific intervals over time to assess for treatment response because new lesions would suggest the need to confirm adherence or consider advancing therapy. 
may or may not be covered by insurance, and there are substantial nonmedical costs of MS, including loss of income, reduced productivity, modifications required for the home and car, and, potentially, a lowered quality of life for the patient. ${ }^{38}$ In individuals who have had MS for many years, the benefit of continuing a DMT is less defined, although there is a paucity of data regarding treatment cessation in this population.

\section{Discussion}

MS is a potentially devastating lifelong disease. It is the most common cause of nontraumatic disability in young adults in the United States. It can limit physical and mental function, and it can be financially burdensome for people with MS and their families. MS can affect relationships, employment, and independence.

The Framework Taskforce emphasizes numerous recommendations and practical strategies in managing the health and wellness of people with MS. In addition to discussing MS disease characteristics and DMTs, this article has reviewed how to select therapies, when to consider switching, and how to manage a suboptimal treatment response. Treating the disease at an early stage, within the first 5 years of clinical symptom onset, is an important consideration. Magnetic resonance imaging is recommended before starting medication or switching DMTs. As discussed, new lesions suggest the need to confirm adherence or consider advancing therapy. Best practice algorithms and shared decision-making strategies have been presented.

The symptoms of MS are complex and can change frequently, resulting in uncertainty, which, in turn, may impair quality of life for people with MS. Clinicians need to be hopeful yet honest and authentic with their patients. Although the use of DMTs is very important, the importance of taking a multidisciplinary approach to the comprehensive care of the person with MS cannot be overstated. This approach requires a team of qualified individuals who often do not exist within the same walls, so effective communication between specialties (or even health systems) is essential in the dynamic framework of MS care.

Acknowledgments: This continuing education article is based on information from the Framework of Care consensus conference, which was organized as a special project by the CMSC. We thank Lori Saslow (Great Neck, NY), a medical writing consultant on behalf of the CMSC, for her assistance with manuscript development, preparation, and editing. Ms. Saslow's work was funded by the CMSC.
Financial Disclosures: Dr. Newsome has served on scientific advisory boards for Biogen, Genentech, Novartis, and Genzyme, and has performed contracted research (institution received funds) for Biogen, Genentech, and Novartis. Dr. Aliotta has served on speakers' bureaus for Astellas Pharma, Actavis, Augmenix, and Allergan and has performed contracted research for Allergan. Dr. Bainbridge has disclosed no relevant financial relationships. Dr. Bennett has served on speakers' bureaus for Acorda Therapeutics, Biogen, and Medtronic; has received consulting fees from and performed contracted research for Acorda Therapeutics; and is chair of the Clinical Events Committee at Innovative Technologies. Dr. Cutter has participated on Data and Safety Monitoring Committees for AMO Pharma, Apotek, Gilead Pharmaceuticals, Horizon Pharmaceuticals, Modigenetech/ Prolor, Merck, Merck/Pfizer, Opko Biologics, Neuren, Sanofi-Aventis, Reata Pharmaceuticals, Receptos/Celgene, Teva Pharmaceuticals, NHLBI (Protocol Review Committee), and NICHD (OPRU Oversight Committee); has received consulting fees from and/or served on speakers' bureaus and scientific advisory boards for Cerespir, Genzyme, Genentech, Innate Therapeutics, Janssen Pharmaceuticals, Klein-Buendel Incorporated, Medlmmune, Medday, Nivalis, Novartis, Opexa Therapeutics, Roche, Savara, Somahlution, Teva Pharmaceuticals, Transparency Life Sciences, and TG Therapeutics; and is President of Pythagoras, Inc., a private consulting company located in Birmingham, AL. Ms. Fenton has disclosed no relevant financial relationships. Dr. Lublin has received consulting fees/fees for nonCME/CE activities from Bayer HealthCare Pharmaceuticals, Biogen, EMD Serono, Novartis, Teva Neuroscience, Actelion, Sanofi/Genzyme, Acorda, Questcor/Mallinckrodt, Roche/Genentech, Medlmmune, Osmotica, Xenoport, Receptos/Celgene, Forward Pharma, Akros, TG Therapeutics, AbbVie, Toyama, Amgen, Medday, Atara Biotherapeutics, Polypharma, Pfizer, Johnson \& Johnson, Revalesio, Coronado Bioscience, and Bristol-Myers Squibb; has served on speakers' bureaus for Genentech/Roche and Genzyme/Sanofi; has performed contracted research for Acorda, Biogen, Novartis, Teva Neuroscience, Genzyme, Xenoport, and Receptos; is the co-chief editor of Multiple Sclerosis and Related Disorders; and has an ownership interest in Cognition Pharmaceuticals. Ms. Northrop has disclosed no relevant financial relationships. Dr. Rintell has received consulting fees from Novartis and has served as a patient education speaker for Teva Neuroscience. He started as a salaried employee of Sanofi Genzyme in November 2015. Dr. Rintell's work on this proj ect was completed before he became a salaried employee of Sanofi Genzyme. Mr. Walker has served on scientific advisory boards for EMD Serono and Sanofi Genzyme and owns stock in Biogen. Dr. Weigel has received consulting fees from Mallinckrodt, Genzyme, and Genentech, and has served on speakers' bureaus for Bayer Corp, Acorda Therapeutics, Teva Neuroscience, Biogen, Mallinckrodt, Genzyme, Novartis, and Pfizer. Dr. Zackowski has performed contracted research for Acorda Therapeutics. Dr. Jones has received consulting fees from Biogen and Novartis, and has performed contracted research for Biogen.

Funding/Support: Funding for the Framework of Care consensus conference was provided by the CMSC, Mallinckrodt Pharmaceuticals, and Mylan Pharmaceuticals.

\section{References}

1. Halper J, Harris C. Nursing Practice in Multiple Sclerosis: A Core Curriculum. 3rd ed. New York, NY: Springer Publishing Co; 2012.

2. Whetten-Goldstein K, Sloan FA, Goldstein LB, Kulas ED. A comprehensive assessment of the cost of multiple sclerosis in the United States. Mult Scler. 1998;4:419-425. 
3. Halper J, Burks JS. Care patterns in multiple sclerosis: principal care, comprehensive team care, consortium care. NeuroRehabilitation. 1994;4:67-75

4. Costello K, Halper J, Kalb R, Skutnik L, Rapp R. The use of diseasemodifying therapies in multiple sclerosis: principles and current evidence. http://www.nationalmssociety.org/getmedia/5ca284d3-fc7c4ba5-b005-ab537d495c3c/DMT_Consensus_MS_Coalition_color. Published March 2015. Accessed April 20, 2015.

5. Emerging Therapies Collaborative. Medications. http://www.mscoalition.org/emergingtherapies/medications. Accessed April 20, 2015.

6. National Multiple Sclerosis Society. Medications. http://www.nationalmssociety.org/Treating-MS/Medications. Published 2016. Accessed August 10, 2016.

7. Rae-Grant A, Bennett A, Sanders AE, Phipps M, Cheng E, Bever C. Quality improvement in neurology: multiple sclerosis quality measures: executive summary. Neurology. 2015;85:1904-1908.

8. Lublin FD, Reingold SC; National Multiple Sclerosis Society (USA) Advisory Committee on Clinical Trials of New Agents in Multiple Sclerosis. Defining the clinical course of multiple sclerosis: results of an international survey. Neurology. 1996;46:907-911.

9. Lublin FD, Reingold SC, Cohen JA, et al. Defining the clinical course of multiple sclerosis: the 2013 revisions. Neurology. 2014;83:278-286.

10. Lublin FD, Baier M, Cutter $G$. Effect of relapses on development of residual deficit in multiple sclerosis. Neurology. 2003;61:1528-1532.

11. Fox RJ. Relapse management in multiple sclerosis. In: Rae-Grant $A D$, Fox R, Bethoux F, eds. Multiple Sclerosis and Related Disorders. New York, NY: Demos Medical Publishing; 2013:94-100.

12. Scott TF, Schramke CJ. Poor recovery after the first two attacks of multiple sclerosis is associated with poor outcome five years later. J Neurol Sci. 2010;292:52-56.

13. Scott TF, Schramke CJ, Novero J, Chieffe C. Short-term prognosis in early relapsing-remitting multiple sclerosis. Neurology. 2000; 55:689-693.

14. O'Riordan Jl, Thompson AJ, Kingsley DP, et al. The prognostic value of brain MRI in clinically isolated syndromes of the CNS: a 10-year follow-up. Brain J Neurol. 1998; 121 (pt 3):495-503.

15. Okuda DT, Siva A, Kantarci O, et al. Radiologically isolated syndrome: 5-year risk for an initial clinical event. PLoS One. 2014; 9:e90509.

16. Freedman MS, Selchen D, Arnold DL, et al. Treatment optimization in MS: Canadian MS Working Group updated recommendations. Can J Neurol Sci.2013:40:307-323.

17. American Academy of Neurology. Position statement: availability of disease modifying therapies (DMT) for treatment of relapsing forms of multiple sclerosis. https://www.aan.com/uploadedFiles/ Website_Library_Assets/Documents/6.Public_Policy/1.Stay_ Informed/2.Position Statements/DiseaseModTheraM̄S PosStatement. pdf. Accessed April $\overline{1}, 2015$.

18. Tintore M, Rovira Á, Otero-Romero $S$, et al. Factors that determine disease course: early changes contribute to predict long-term prognosis: the "Barcelona inception cohort." Paper presented at: Joint ACTRIMSECTRIMS meeting; September 2014; Boston, MA. Abstract PS9.4.

19. Ford C; Therapeutic Decision Making Consensus Group. Therapeutic approach for patients with aggressive onset or poor prognostic indicators in MS. Int J MS Care. 2014;16(suppl 6): 18-22.

20. Plavina $T$, Subramanyam $M$, Bloomgren $G$, et al. Anti-JC virus antibody levels in serum or plasma further define risk of natalizumabassociated progressive multifocal leukoencephalopathy. Ann Neurol. 2014;76:802-812.
21. Traboulsee A, Simon JH, Stone L, et al. Revised recommendations of the Consortium of MS Centers Task Force for a standardized MRI protocol and clinical guidelines for the diagnosis and follow-up of multiple sclerosis. AJNR Am J Neuroradiol. 2016;37:394-401.

22. Traboulsee A, Li D. Addressing concerns regarding the use of gadolinium in a standardized MRI protocol for the diagnosis and follow-up of multiple sclerosis [published online ahead of print September 1 , 2016]. AJNR Am J Neuroradiol. doi:10.3174/ajnr.A4943.

23. Stangel M, Penner IK, Kallmann BA, Lukas C, Kieseier BC. Towards the implementation of "no evidence of disease activity" in multiple sclerosis treatment: the multiple sclerosis decision model. Ther Adv Neurol Disord. 2015;8:3-13.

24. Bevan CJ, Cree BAC. Disease activity free status: a new end point for a new era in multiple sclerosis clinical research? JAMA Neurol. 2014;71:269-270.

25. Polman $\mathrm{CH}, \mathrm{O}^{\prime}$ Connor PW, Havrdova $\mathrm{E}$, et al. A randomized, placebo-controlled trial of natalizumab for relapsing multiple sclerosis. $\mathrm{N}$ Engl J Med. 2006;354:899-910.

26. Kappos L, Radue E-W, O'Connor $\mathrm{P}$, et al. A placebo-controlled trial of oral fingolimod in relapsing multiple sclerosis. N Engl J Med. 2010;362:387-401.

27. Gold R, Kappos L, Arnold DL, et al. Placebo-controlled phase 3 study of oral BG-12 for relapsing multiple sclerosis. N Engl J Med. 2012;367:1098-1107.

28. Calabresi PA, Kieseier BC, Arnold DL, et al. Pegylated interferon $\beta$-1a for relapsing-remitting multiple sclerosis (ADVANCE): a randomised, phase 3, double-blind study. Lancet Neurol. 2014;13:657-665.

29. Coles AJ, Twyman CL, Arnold DL, et al. Alemtuzumab for patients with relapsing multiple sclerosis after disease-modifying therapy: a randomised controlled phase 3 trial. Lancet. 2012;380:1829-1839.

30. Prosperini L, Gallo V, Petsas N, Borriello G, Pozzilli C. One-year MRI scan predicts clinical response to interferon beta in multiple sclerosis. Eur J Neurol. 2009; 16:1202-1209.

31. Zecca C, Disanto G, Sormani MP, et al. Relevance of asymptom atic spinal MRI lesions in patients with multiple sclerosis. Mult Scler. 2016;22:782-791.

32. He A, Spelman T, Jokubaitis V, et al. Comparison of switch to fingolimod or interferon beta/glatiramer acetate in active multiple sclerosis. JAMA Neurol. 2015;72:405-413.

33. Kalincik T, Horakova D, Spelman T, et al. Switch to natalizumab versus fingolimod in active relapsing-remitting multiple sclerosis. Ann Neurol. 2015;77:425-435.

34. Coyle PK, Foley JF, Fox EJ, Jeffery DR, Munschauer FE, Tornatore C. Best practice recommendations for the selection and management of patients with multiple sclerosis receiving natalizumab therapy. Mult Scler. 2009; 15(suppl):S26-S36.

35. Coyle PK. Switching therapies in multiple sclerosis. CNS Drugs. 2013; 27:239-247.

36. Tornatore C, Phillips JT, Khan O, Miller A, Ally A. Consensus opinion of US neurologists on practice patterns in radiologically and clinically isolated syndrome and relapsing-remitting MS. Paper presented at: Joint ACTRIMS-ECTRIMS meeting; September 2014; Boston, MA. Poster 295

37. Arnold DL, Campagnolo D, Panitch $\mathrm{H}$, et al. Glatiramer acetate after mitoxantrone induction improves MRI markers of lesion volume and permanent tissue injury in MS. J Neurol. 2008;255:1473-1478.

38. Brandes D, Rieckmann P. The manifold economic impact of multiple sclerosis: indirect and direct costs of managing patients. Eur Neurol Rev. 2012;7(suppl 2):17-23. 